\title{
The Mechanism of Soft Nanoparticle Diffusion in Entangled Polymer Melts
}

Umesh M Shrestha ${ }^{1}$, Lu Han, ${ }^{2}$ Tomonori Saito, ${ }^{2}$ Kenneth S. Schweizer, ${ }^{3-5}$ and Mark D.

Dadmun $^{1,2}$

${ }^{1}$ Department of Chemistry, University of Tennessee, Knoxville, TN 37996, USA

${ }^{2}$ Chemical Sciences Division, Oak Ridge National Laboratory, Oak Ridge, TN 37831, USA

${ }^{3}$ Department of Materials Science, University of Illinois, Urbana, IL 61801, USA

${ }^{4}$ Department of Chemistry, University of Illinois, Urbana, IL 61801, USA

${ }^{5}$ Materials Research Laboratory, University of Illinois, Urbana, IL 61801, USA

corresponding authors: dad@utk.edu, kschweiz@illinois.edu 


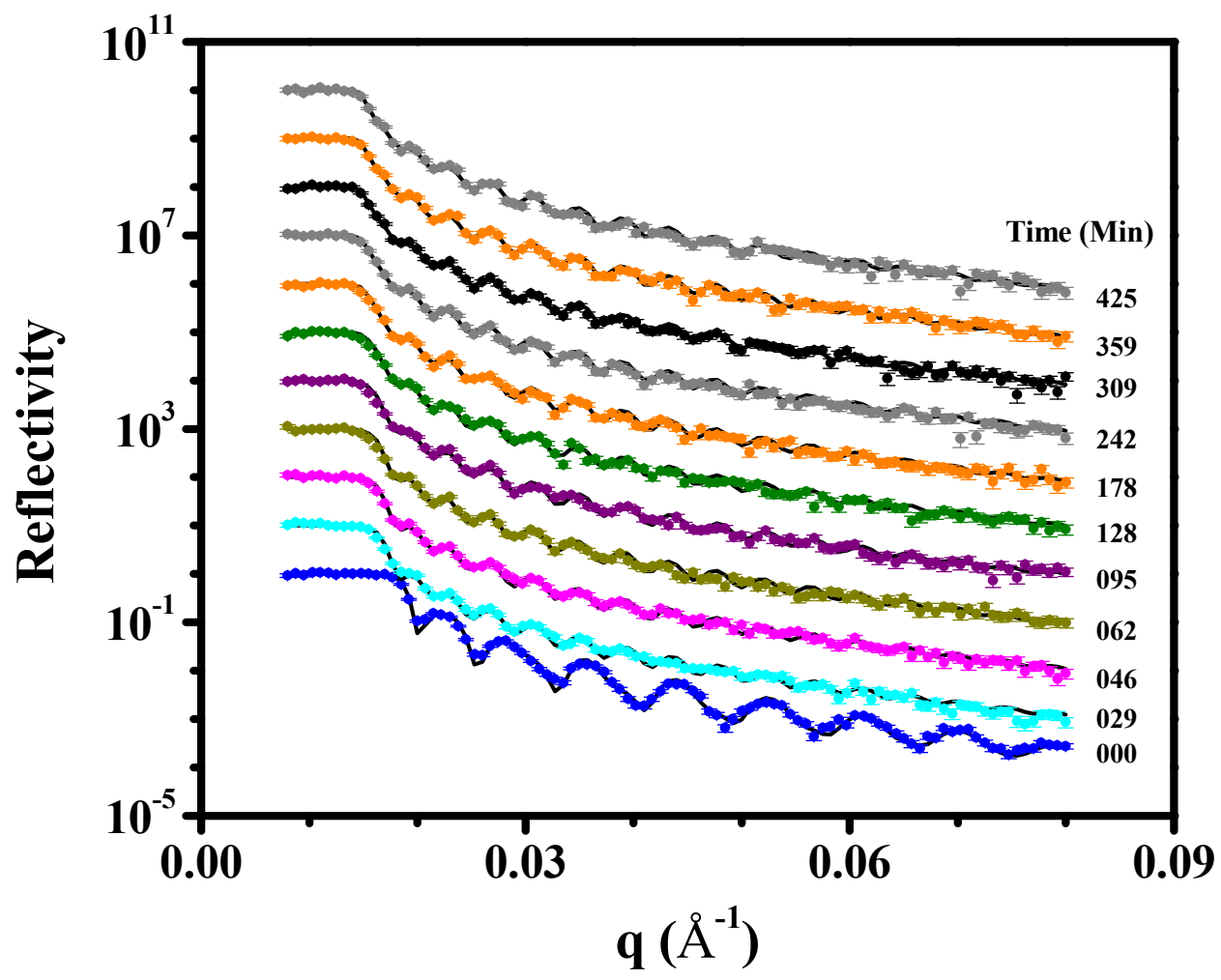

Figure S1: Representative reflectivity profile of the NP1-dPS bilayer for as cast film and annealed at different annealing times. Symbols correspond to the experimental data and the solid line passing through the symbols is the best fit. The data have been shifted vertically for clarity.

In Figure S1, the reflectivity profiles are shifted vertically for clarity. The symbols are experimental data and the solid line through them show the best fit. The bottom curve is for the bilayer film at $90^{\circ} \mathrm{C}$ which is below $\mathrm{T}_{\mathrm{g}}$ of both NP and d-PS layer and considered an as-cast film. Although multiple reflectivity profiles were measured at $130^{\circ} \mathrm{C}$ and different annealing times, only a few profiles are shown for clarity. The annealing times are shown along with the reflectivity profile. Figure S2 shows the change in scattering length density profile corresponding to the fits in Figure S1. 


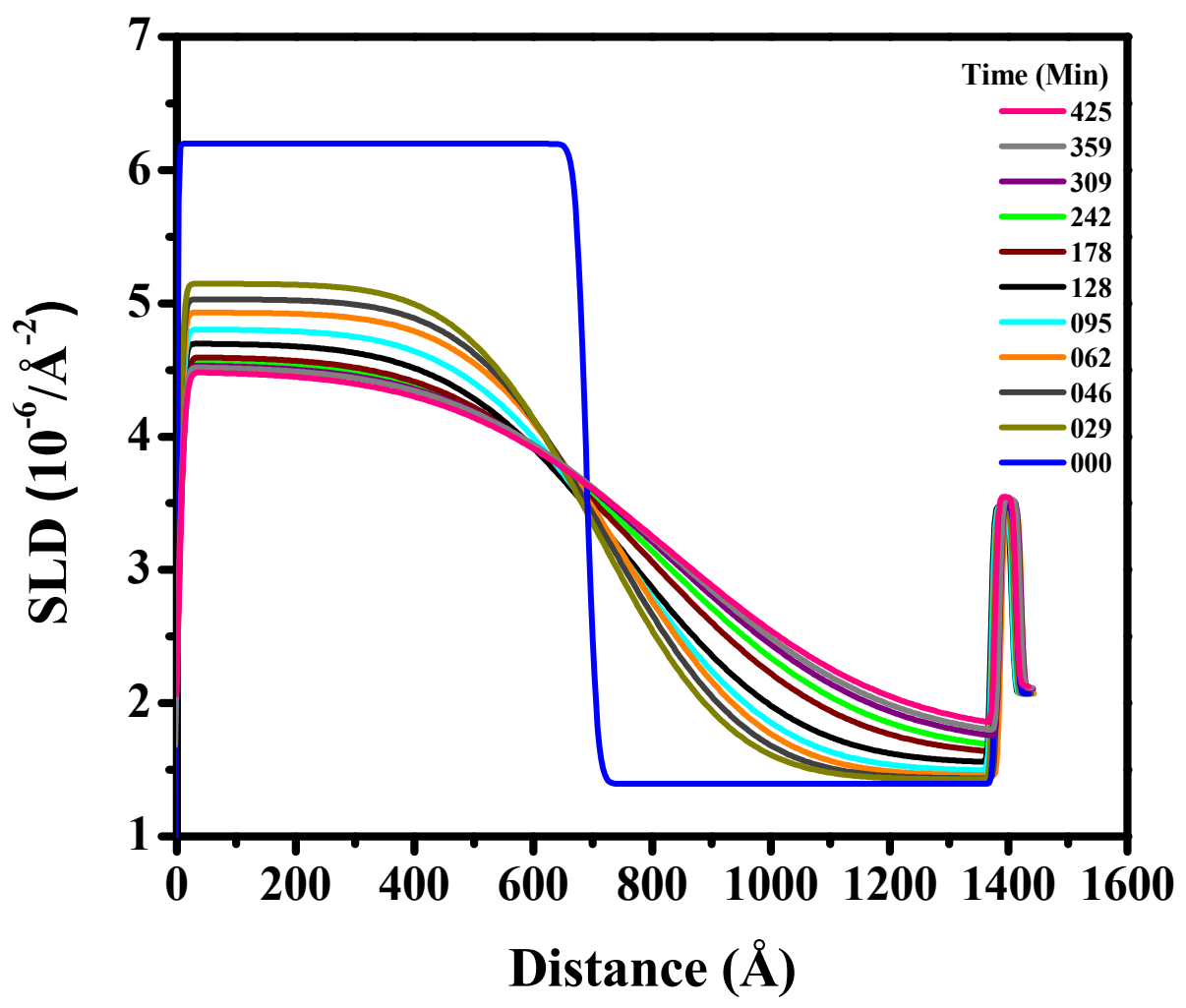

FigureS2: The scattering length density profile of NP1-dPS extracted from the best fit of the reflectivity profile.

Figure S3 shows the change in volume fraction profile corresponding to the SLD profiles in Figure S2 and fits in Figure S1. 


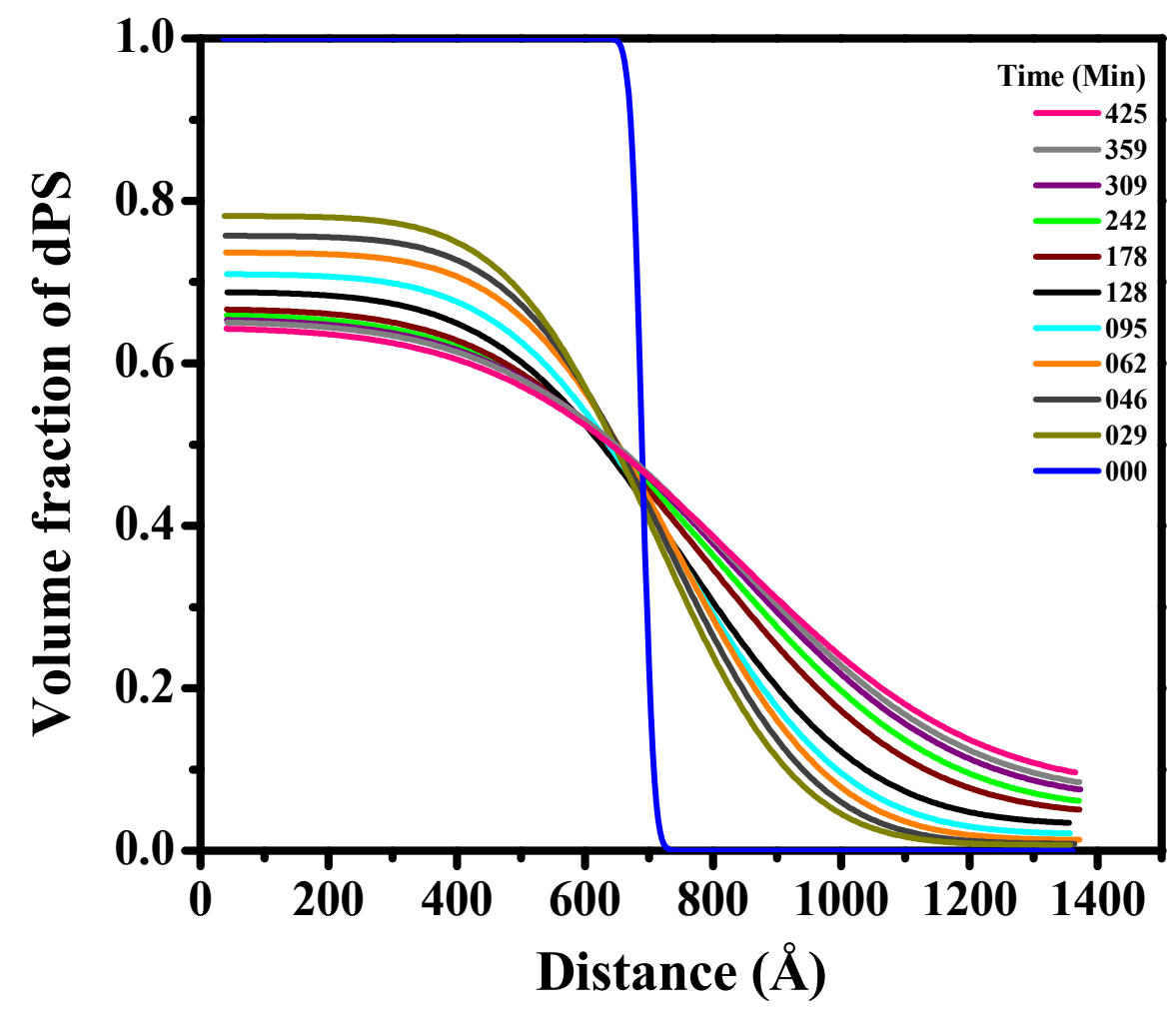

Figure S3: The volume fraction profiles of the NP1-dPS bilayer as cast and annealed for different time.

In Figure S4, the data shows that at the early state of annealing, the interfacial width changes with time as $\mathrm{t}^{1 / 4}$ indicating local dynamics, while at longer annealing times, the variation in the interfacial width changes to a Fickian $\mathrm{t}^{1 / 2}$ dependence. In the NP1 bilayer, the transition from sub-diffusive to diffusive behavior occurs at ca. 177 mins of annealing. 


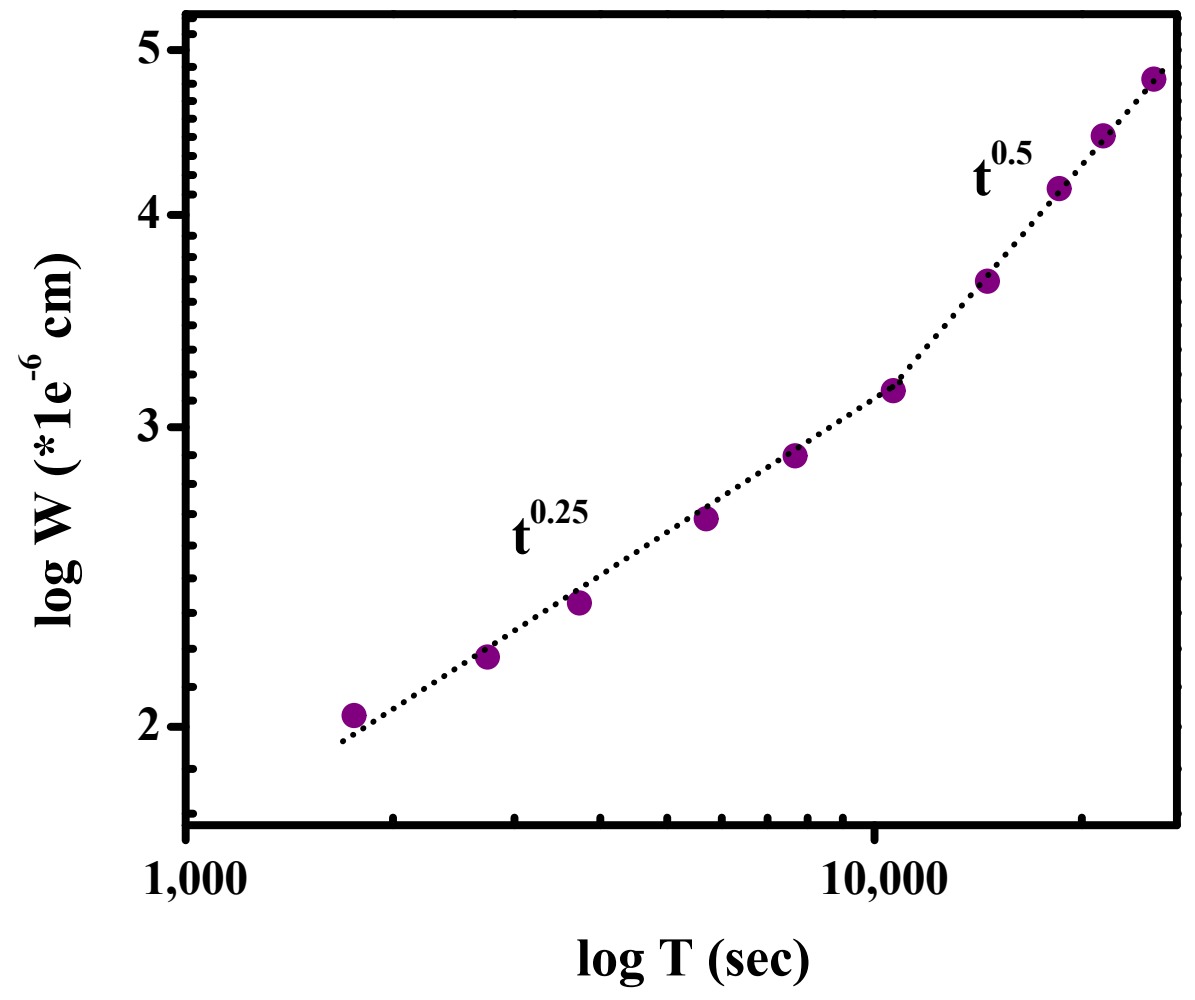

Figure S4: log-log plot of interfacial width vs annealing time for NP1-dPS bilayer. The data fits for the early annealing time regime to a dependence of $t^{1 / 4}$. The later annealing time regime follows the Fickian $t^{1 / 2}$ behavior. 

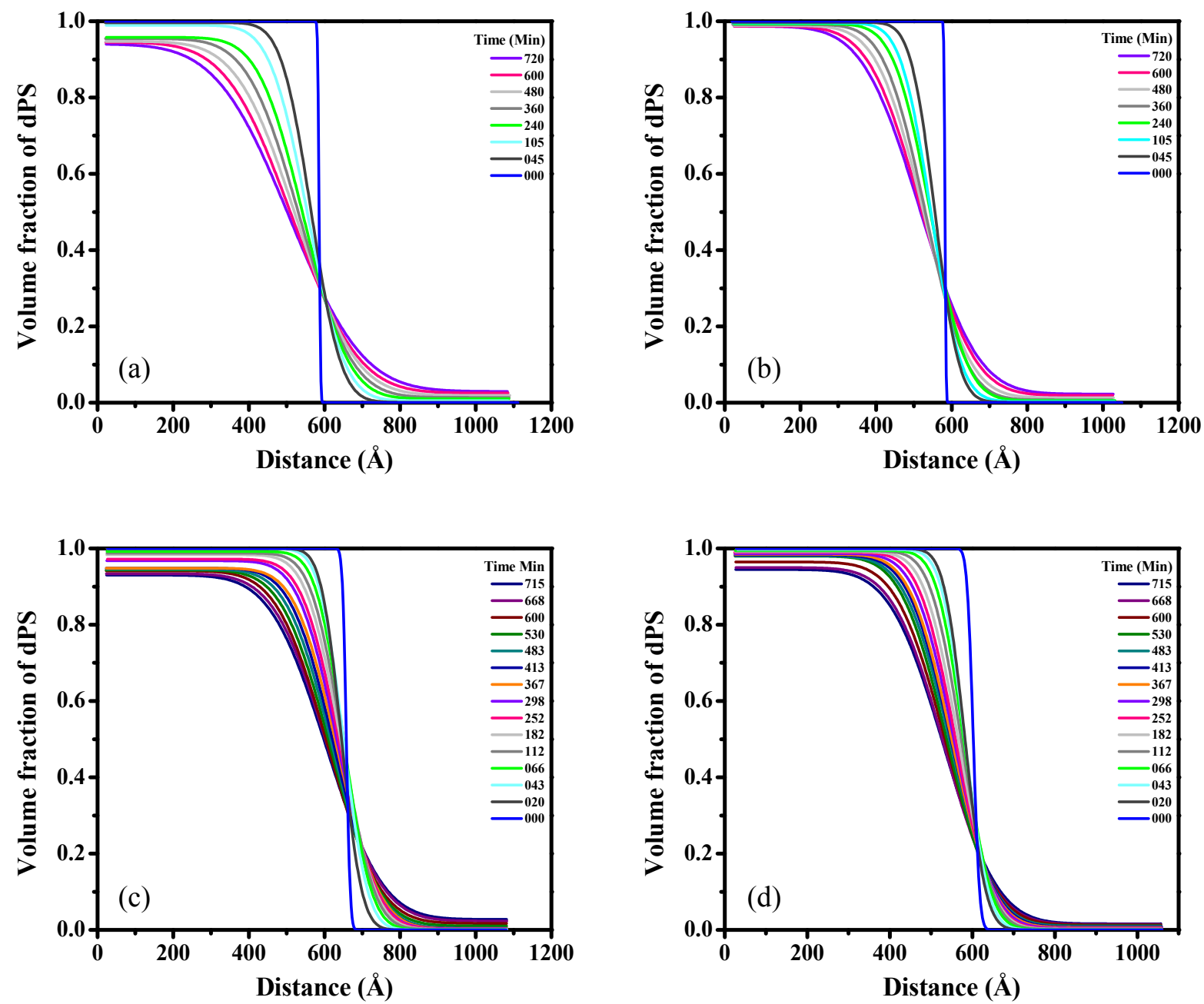

Figure S5: The volume fraction profiles of the NP-dPS bilayer as cast and annealed for different annealing times for $M_{w}$ of NPs (a) 238k (NP2), (b) 600k (NP10), (c) 854k (NP50) and (d) 1189k (NP20) respectively. 
Figure S6 shows that, independent of the $\mathrm{M}_{\mathrm{w}}$ of the SNPs, for the early stage of annealing the interfacial width changes as $t^{1 / 4}$. After extended annealing, this changes to $t^{1 / 2}$ indicating the dynamics changes from sub-diffusive to diffusive motion for all bilayers. However, the time needed to transition from sub-diffusive to diffusive motion increases from $\sim 177$ mins to $\sim 430$ mins with an increase in $\mathrm{M}_{\mathrm{w}}$ of the NP.


Figure S6: log-log plot of interfacial width vs annealing time for (a) NP2, (b) NP10, (c) NP20 and (d) NP50, respectively. 


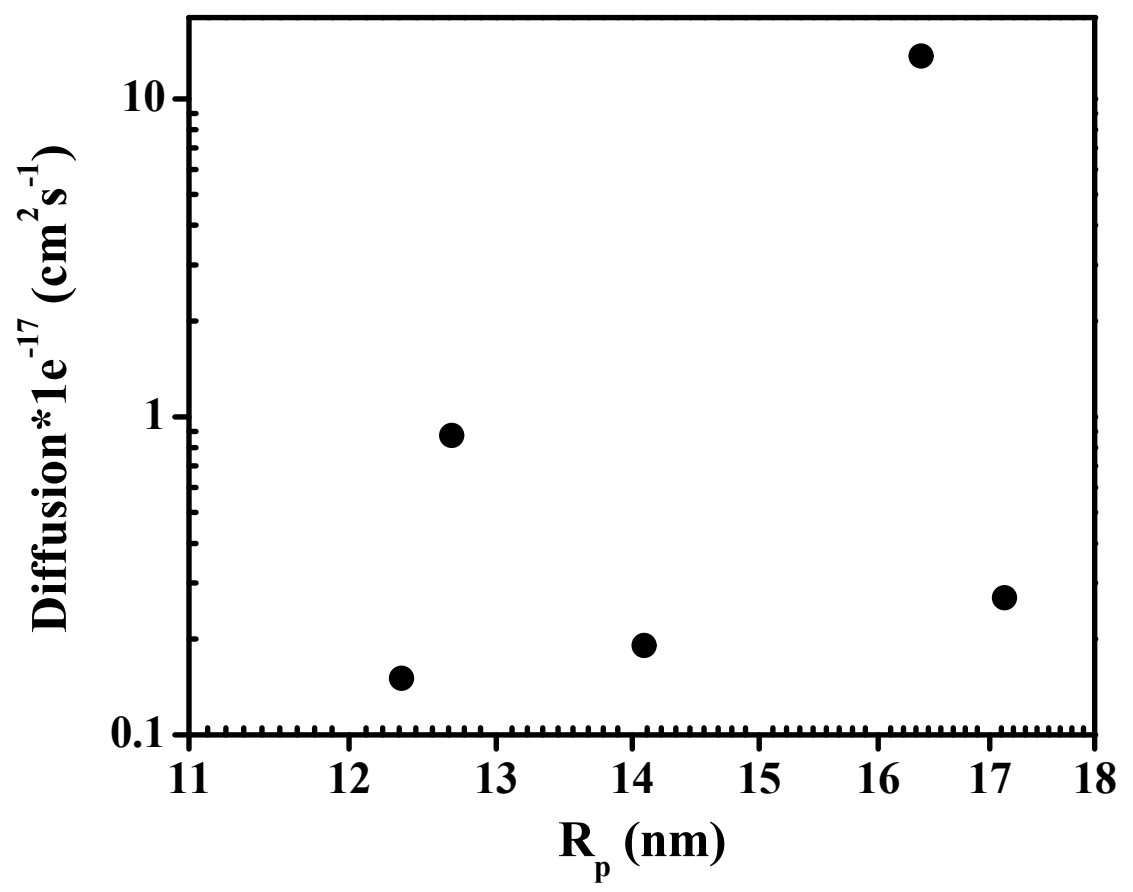

Figure S7: The tracer diffusion coefficient of polystyrene nanoparticles in Table 1 of the main manuscript as a function of nanoparticle diameter.

Figure S7 presents the change in tracer diffusion coefficient of the SNPs in Table 1 as a function of particle radius. As discussed in the main text, the diffusion coefficient of NP1 ( 13.6 $\mathrm{x} 10^{-17} \mathrm{~cm}^{2} / \mathrm{s}$ ) differs by an order of magnitude from the other particles, which is ascribed to its low molecular weight and crosslink density which qualitatively changes it geometric nature. 